\title{
Size Effect on Sensitivity to External Pressure and Caloric Effects in TGS: Ceramics and Nanocomposites
}

\author{
E.A. Mikhaleva ${ }^{1}$, I.N. Flerov ${ }^{1,2, *}$, E.V. Bogdanov ${ }^{1,3}$, V.S. Bondarev ${ }^{1,2}$, M.V. Gorev ${ }^{1,2}$, E. \\ Rysiakiewicz-Pasek ${ }^{4}$
}

${ }^{1}$ Kirensky Institute of Physics, Krasnoyarsk Scientific Center, Siberian Branch, Russian Academy of Sciences, 660036 Krasnoyarsk, Russia

${ }^{2}$ Institute of Engineering Physics and Radio Electronics, Siberian Federal University, 660041 Krasnoyarsk, Russia

${ }^{3}$ Institute of Engineering Systems and Energy, Krasnoyarsk State Agrarian University, 660049 Krasnoyarsk, Russia

${ }^{4}$ Department of Experimental Physics, Wroclaw University of Science and Technology, 50-370 Wroclaw, Poland

\begin{abstract}
Experimental study of temperature-pressure phase diagrams revealed a slight difference in the sensitivity to hydrostatic pressure of triglycine sulfate (TGS) as a bulk (single crystal, ceramics), and nanoparticles embedded in porous glass (TGS+PG). An analysis of the pressure sensitivity, $\mathrm{dT} / \mathrm{p}$ and $\mathrm{dT} / \mathrm{d} \sigma$, and entropies of the $\mathrm{P} 2{ }_{1} \leftrightarrow \mathrm{P} 2{ }_{1} / \mathrm{m}$ phase transition showed the closeness of the barocaloric parameters in various materials under study. Due to the strong anisotropy of thermal expansion, the piezocaloric effect under pressure along the axis $c$ exceeds the barocaloric effect in single-crystal TGS. The
\end{abstract}


reasons for the strong reduction in the electrocaloric effect in the ferroelectric component of nanocomposites compared with a single-crystal are discussed.

Keywords: Ferroelectric nanocomposite, Phase transition, High pressure, Caloric Effects

Corresponding author. Kirensky Institute of Physics, Krasnoyarsk Scientific Center, Siberian Branch, Russian Academy of Sciences, 660036 Krasnoyarsk, Russia E-mail address: flerov@iph.krasn.ru (I.N.Flerov)

\begin{abstract}
Abbreviations:
CE - caloric effect; TGS - triglycine sulfate; ECE - electrocaloric effect; MCE magnetocaloric effect; $\mathrm{BCE}$ - barocaloric effect; PCE - piezocaloric effect.
\end{abstract}




\section{Introduction}

Materials, in particular ferroics, undergoing phase transitions accompanied by a large change in entropy are considered as promising solid-state refrigerants for use in alternative cooling cycles that are competitive with traditional gas-compressor refrigerators [1-7]. The reason is that large caloric effects (CEs) can often be realized in these materials, which are associated with a change in such fundamental properties as temperature and entropy under adiabatic and isothermal conditions, respectively, as a result of varying the external field conjugated with the nature of the phase transition. Unlike electro(ECE)- and magneto(MCE)-caloric effects, baro(BCE)- and piezo(PCE)(or elalsto(ElCE) [1])-caloric effects are universal due to the possibility of being implemented in various materials under hydrostatic, $p$, and uniaxial, $\sigma$, pressure. This is due to the fact that the extensive, $\Delta \mathrm{S}_{\mathrm{BCE}}$ and $\Delta \mathrm{S}_{\mathrm{PCE}}$, and intensive, $\Delta \mathrm{T}_{\mathrm{AD}}$, effects are determined mainly by the volumetric or linear thermal expansion of the material: $\Delta \mathrm{S}_{\mathrm{BCE}}=-\int(\partial \mathrm{V} / \partial \mathrm{T})_{\mathrm{p}} \mathrm{dp}, \Delta \mathrm{T}_{\mathrm{AD}}=\int\left(\mathrm{T} / \mathrm{C}_{\mathrm{p}}\right)(\partial \mathrm{V} / \partial \mathrm{T})_{\mathrm{p}} \mathrm{dp}$ and $\Delta \mathrm{S}_{\mathrm{PCE}}=-\int(\partial \mathrm{L} / \partial \mathrm{T})_{\sigma} \mathrm{d} \sigma, \Delta \mathrm{T}_{\mathrm{AD}}=$ $\int\left(\mathrm{T} / \mathrm{C}_{\mathrm{p}}\right)(\partial \mathrm{L} / \partial \mathrm{T})_{\sigma} \mathrm{d} \sigma\left(\mathrm{C}_{\mathrm{p}}\right.$ is the heat capacity) $[2,6,7]$, which is characterized by anomalously large changes in the region of phase transitions, including ferromagnetic, ferroelectric and ferroelastic ones.

Thus, we can talk about the existence of multicaloric properties in one material, such as ferroelectrics $(\mathrm{ECE}+\mathrm{BCE})$ and ferromagnets $(\mathrm{MCE}+\mathrm{BCE})$. In this regard, composite materials, the components of which have a different physical nature, are no less interesting. Moreover, one of the components may not be ferroic, but significantly affect the physical properties of the active ferroid component and, thus, on the caloric effectiveness of the composite. 
Among composites of various types, systems consisting of ferroelectric materials embedded in nanoporous matrices attract the attention of researchers due to the promising possibilities of controlling the physical properties of the active component by varying the pore size. A large number of articles are devoted to the study of the size effect on the permittivity especially in the temperature range of phase transitions in various ferroelectric crystals grown in nanopores [8-15]. Much less attention was paid to the investigation of polarization, heat capacity, thermal expansion and other properties [10,16-18]. Finally, with regard CEs of various nature in ferroelectric nanocomposites, they have not been studied at all.

The most fruitful way to determine the character and degree of influence of restricted geometry on the properties of the active component embedded in a passive matrix is to perform a consistent comparative study of a single crystal, ceramics, and nanocomposite. This approach has been successfully used in the study of ammonium hydrogen sulfate, $\mathrm{NH}_{4} \mathrm{HSO}_{4}$, which undergoes two successive phase transitions of the second and first order, very far from the tricritical point [18]. Of course, it seems important to study a ferroelectric composite with a second order phase transition close to the tricritical point, not accompanied by absorption/release of latent heat and a jump in the parameters and volume of the unit cell, but whose physical properties show strong anomalous behavior. One of the most suitable objects of this kind is triglycine sulfate (TGS) - $\left(\mathrm{CH}_{2} \mathrm{NH}_{2} \mathrm{COOH}\right)_{3} \cdot \mathrm{H}_{2} \mathrm{SO}_{4}$, whose structure and physical properties as a single crystal have been studied in great detail [19-25]. The mechanism of the P $2_{1} \leftrightarrow \mathrm{P} 2{ }_{1} / \mathrm{m}$ phase transition in TGS at $322 \mathrm{~K}$ is related to the ordering/disordering of elementary dipoles associated with reorienting glycine groups and, as a result, the corresponding entropy change is quite large $\Delta \mathrm{S}=0.7 \mathrm{R}$. Recently, we performed studies of the heat 
capacity and thermal expansion of TGS, both prepared in the form of ceramics as well as embedded in nanoporous silicate glasses (TGS+PG) [26]. On the one hand, it was found that the temperature of the $\mathrm{P} 2_{1} \leftrightarrow \mathrm{P} 2{ }_{1} / \mathrm{m}$ phase transition behaves nonmonotonously, increasing slightly and decreasing strongly in composites with pores of $320 \mathrm{~nm}$ (TGS+PG320) and $46 \mathrm{~nm}$ (TGS+PG46), respectively, compared to single crystal and ceramic samples. On the other hand, a decrease in pore size is accompanied by only a small decrease in the entropy of the structural transition, $\leq 10 \%$. The latter circumstance suggests that BCE and PCE in active component of nanocomposites may turn out to be comparable with CEs in bulk samples of TGS (single crystal and ceramics).

In this work, taking into account that the sensitivity of phase transitions to external pressure characterized partially by the shift of the phase transition temperature under hydrostatic, $\mathrm{dT} / \mathrm{dp}$, or uniaxial, $\mathrm{dT} / \mathrm{d} \sigma$, pressure known also as volumetric or linear baric coefficients, is also a very important parameter in the formation of CE [27], we first determined the baric coefficients and then BCE, PCE and ECE in ceramic TGS and nanocomposites TGS+PG320 and TGS+PG46.

\section{Sample preparation and measurement technique}

The samples were prepared using both bulk TGS single-crystal grown from an aqueous solution under equilibrium temperature conditions, and porous silicate glasses thermal properties of which were characterized in recent studies [18]. A quasi-ceramic discshaped pellets of $8 \mathrm{~mm}$ diameter and $1.3 \mathrm{~mm}$ thickness were prepared by pressing of crushed TGS without high-temperature heat treatment. To prepare TGS+PG320 and TGS+PG46 nanocomposites, dry empty glasses (with porosity about 55\% in PG46 and $50 \%$ in PG320) in the form of rectangular plates with dimensions of $1.0 \times 1.0 \times 0.05$ 
$\mathrm{cm}^{3}$ were placed in a saturated aqueous solution of TGS at a temperature of $70{ }^{\circ} \mathrm{C}$ for about 20 minutes After thorough drying at $100^{\circ} \mathrm{C}$, the surfaces of the filled glasses were cleaned by mechanical polishing. This procedure was repeated 5-8 times. The degree of filling of glass matrices was estimated as the ratio between the volumes of embedded TGS and matrices: $26 \%$ for TGS+PG46 and 29.5\% for TGS+PG320, which corresponds to the following ratios between the volumes of TGS and pores: $47 \%$ and $56 \%$, respectively. For dielectric measurements, silver electrodes were deposited on the surface of the sample.

Since the crystallographic parameters in both phases of TGS were determined repeatedly [23-25],the samples under study were characterized only at room temperature, i.e. in the ferroelectric monoclinic $\mathrm{P} 2{ }_{1} / \mathrm{m}$ phase, by XRD using a Bruker D8 ADVANCE powder diffractometer ( $\mathrm{Cu}-\mathrm{Ka}$ radiation). In accordance with the results of Rietveld refinement, no additional phases were observed in TGS, TGS + PG46 and TGS + PG320 (Fig.1)

Table 1 shows the unit cell parameters of all the studied samples in comparison with those for single-crystal TGS [24]. The influence of the size factor turned out to be insignificant and most noticeably manifested in a slight decrease in the unit cell volume.

Table 1. Unit cell parameters in the $\mathrm{P} 2{ }_{1}$ phase of a ferroelectric TGS in the form of quasi-ceramics and embedded in PG in comparison with the parameters of a single crystal [24].

\begin{tabular}{|l|l|l|l|l|l|}
\hline & $\mathrm{a}(\mathrm{nm})$ & $\mathrm{b}(\mathrm{nm})$ & $\mathrm{c}(\mathrm{nm})$ & $\beta\left(^{\circ}\right)$ & $\mathrm{V}\left(\mathrm{nm}^{3}\right)$ \\
\hline Single crystal & $0.94276(5)$ & $1.26603(6)$ & $0.57423(3)$ & $110.335(2)$ & $0.64267(2)$ \\
\hline Quasi-ceramics & $0.94274(5)$ & $1.26592(5)$ & $0.57408(3)$ & $110.338(2)$ & $0.64242(6)$ \\
\hline
\end{tabular}




\begin{tabular}{|l|l|l|l|l|l|}
\hline TGS+PG320 & $0.94216(9)$ & $1.26586(9)$ & $0.57348(5)$ & $110.318(5)$ & $0.64141(9)$ \\
\hline TGS+PG46 & $0.94183(9)$ & $1.26522(9)$ & $0.57358(5)$ & $110.329(5)$ & $0.64092(9)$ \\
\hline
\end{tabular}

The size of ferroelectric nanoparticles, $d_{\text {cryst, }}$ grown in the matrix pores was determined using the TOPAS 4.2 program [28]. It was found that this parameter is smaller and larger than the pore size in PG320 $\left(\mathrm{d}_{\text {cryst }}=160 \mathrm{~nm}\right)$ and PG46 $\left(\mathrm{d}_{\text {cryst }}=74\right.$ $\mathrm{nm})$, respectively. The excess of crystallite size over pore size observed TGS+PG46 can be explained as related to the formation of dendritic clusters in the pores $[18,29]$.

The size effect on sensitivity to hydrostatic pressure both of TGS prepared as quasi-ceramics and embedded in PG was studied using a home-made high-pressure installation. The sample with electrodes was placed in a holder inside a piston-cylinder type chamber associated with a pressure multiplier filled with oil from the low pressure side created by an external pumping station. The ratio between the sections of low and high pressure plungers made it possible to realize a pressure in the measuring chamber of up to $0.5 \mathrm{GPa}$. Pentane was used as a pressure transmitting medium due to its optimal electrical and thermal conductivity, and viscosity. Moreover, unlike oil, pentane, penetrated into ceramic and composite samples, could be easily removed and samples could be used in other experiments. Pressure dependences of the phase transition temperature, $\mathrm{T}_{0}(\mathrm{p})$, were investigated by measuring the permittivity at frequency $1 \mathrm{kHz}$ using an E7-20 immittance meter. The experiments were carried out in the pressure range of $0-0.4 \mathrm{GPa}$, which was controlled using a manganin gauge with an error of about $\pm 10^{-3} \mathrm{GPa}$. The temperature was controlled with the inaccuracy of about $\pm 0.3 \mathrm{~K}$ using a copper-constantan thermocouple attached to the sample. To ensure the reliability of the results, the measurements under both increasing and decreasing pressure cycles were performed. 
In addition, the ferroelectric properties and the heat capacity of the TGS+PG320 nanocomposite in a home-made adiabatic calorimeter $[21,30]$ were also studied. The high vacuum $\left(10^{-6}\right.$ Torr $)$ in the measuring chamber made it possible to exclude the presence of water absorbed by the sample.

Calorimetric measurements were carried out using discrete as well as continuous heating. In the former case, the calorimetric step was varied from 1.0 to $3.0 \mathrm{~K}$. In the latter case, the sample under study was heated at rates of $\mathrm{dT} / \mathrm{dt}=0.15-0.30 \mathrm{~K} / \mathrm{min}$. The inaccuracy in the heat capacity determination did not exceed $1.0-1.5 \%$.

The temperature dependences of the permittivity, $\varepsilon(T)$, were investigated as described above in the temperature range $150-350 \mathrm{~K}$ at frequencies of $1 \mathrm{kHz}$ and 1 MHz. Continuous heating was used with a rate of $(0.2-1.0) \mathrm{K} / \mathrm{min}$.

Direct measurements of intensive ECE were carried out under adiabatic conditions by means of a technique previously used to study ECE of a TGS single crystal under equalibrium and nonequilibrium conditions [21]. A platinum resistance thermometer attached to the adiabatic screen closest to the sample allowed us to monitor the temperature of the sample with high-precision. The temperature difference between the thermometer and the sample was monitored by a doubled copper-constantan thermocouple whose output was supplied to the automatic control circuit.

BCE and PCE were determined using both $\mathrm{T}-\mathrm{p}$ and $\mathrm{T}-\sigma$ phase diagrams, and data on the heat capacity and thermal expansion of single crystal, quasi-ceramics and nanocomposites studied earlier [19-21, 26] and in this paper. To this end, we used the previously developed approach [31], which was successfully used in the analysis of the baro- and piezocaloric efficiency of various ferroid materials [7,27,32,33].

\section{Results and Discussion}


The temperature dependences of the permittivity of quasi-ceramics and nanocomposites TGS + PG46 and TGS + PG320 measured at a frequency $1 \mathrm{kHz}$ under pressure in the range $0-0.4 \mathrm{GPa}$ are presented in Fig. 2.

Comparison of the data for single-crystal [34] and quasi-ceramics shows that in the latter material, placed in pentane as a pressure transmitting medium, the maximum value of the anomalous part of the permittivity, $\Delta \varepsilon=25$, at $\mathrm{T}_{0}$ and $\mathrm{p}=0$ is a thousand times smaller (Fig.2a). A stronger decrease in the permittivity peak was observed in both composites, $\Delta \varepsilon \leq 1$.

A decrease in the anomalous dielectric constant under pressure detected in all samples under study was also observed earlier for single-crystal TGS [20,35,36]. One can think that one of the reasons for this phenomenon is related to the approach of the $\mathrm{P} 2{ }_{1} \leftrightarrow \mathrm{P} 21 / \mathrm{m}$ phase transition to the triple point in the $\mathrm{T}-\mathrm{p}$ phase diagram of the TGS [20].

Figures $2 \mathrm{~b}$ and $2 \mathrm{c}$ also show a stronger suppression of the maximum value $\Delta \varepsilon$ in nanocomposites even at very low pressure. One can assume that this feature is due to the structure of the TGS+PG capacitor, which consists of two dissimilar parts that interact with each other only mechanically.

Nevertheless, it is obvious that the phase transition temperature increases under pressure in all samples under study (Fig. 2d). The shift of the permittivity peak is linear and is characterized by the baric coefficients presented in Table 2 . The values of $\mathrm{dT}_{0} / \mathrm{dp}$ determined by us turned out to be slightly less in comparison with the experimental data for a TGS single-crystal $[20,35,36]$ (Table 2). Thus, the size effect does not significantly affect the sensitivity of ferroelectric TGS to changes in external hydrostatic pressure 
Table 2. Volumetric and linear baric coefficients, and PCE and BCE of single crystal and ceramic TGS as well as nanocomposites TGS+PG320, TGS+PG46. Caloric effects are presented for $\sigma=0.45 \mathrm{GPa}$. Values marked with $*$ refer to the active component in nanocomposites.

\begin{tabular}{|c|c|c|c|c|}
\hline & Single crystal TGS & Ceramics TGS & TGS+PG320 & TGS+PG46 \\
\hline$\left(\mathrm{dT}_{0} / \mathrm{dp}\right)_{\mathrm{exp}}, \mathrm{K} / \mathrm{GPa}$ & $+(26.0 \pm 1.5)[20]$ & $+(23.5 \pm 1.5)$ & $+(19.8 \pm 1.5)$ & $+(19.5 \pm 1.5)$ \\
\hline$\left(\mathrm{dT}_{0} / \mathrm{dp}\right)_{\mathrm{calc}}, \mathrm{K} / \mathrm{GPa}$ & $+(19 \pm 2)$ & $+(63 \pm 7)$ & $+(18 \pm 3)$ & $+(18 \pm 32)$ \\
\hline $\mathrm{dT}_{0} / \mathrm{d} \sigma_{\mathrm{a}}, \mathrm{K} / \mathrm{GPa}$ & $-(5.0 \pm 0.8)$ & & & \\
\hline $\mathrm{dT}_{0} / \mathrm{d} \sigma_{\mathrm{b}}, \mathrm{K} / \mathrm{GPa}$ & $-(15.0 \pm 1.5)$ & & & \\
\hline $\mathrm{dT}_{0} / \mathrm{d} \sigma_{\mathrm{c}}, \mathrm{K} / \mathrm{GPa}$ & $+(39.0 \pm 2.5)$ & & $+(6 \pm 1)$ & $+(6 \pm 1)$ \\
\hline$\left(\mathrm{d} \mathrm{T}_{0} / \mathrm{d} \sigma\right)_{\mathrm{cer}}, \mathrm{K} / \mathrm{GPa}$ & & $+(21 \pm 2)$ & $-(3.3 \pm 0.4)^{*}$ & $-(3.4 \pm 0.4)^{*}$ \\
\hline$\Delta \mathrm{S}_{\mathrm{BCE}}, \mathrm{J} / \mathrm{kg} \cdot \mathrm{K}$ & $-(6.4 \pm 0.4)$ & $-(4.4 \pm 0.3)$ & & \\
\hline$\left(\Delta \mathrm{S}_{\mathrm{PCE}}\right)_{\mathrm{a}}, \mathrm{J} / \mathrm{kg} \cdot \mathrm{K}$ & $+(1.5 \pm 0.2)$ & & & \\
\hline$\left(\Delta \mathrm{S}_{\mathrm{PCE}}\right)_{\mathrm{b}}, \mathrm{J} / \mathrm{kg} \cdot \mathrm{K}$ & $+(4.0 \pm 0.3)$ & & & \\
\hline$\left(\Delta \mathrm{S}_{\mathrm{PCE}}\right)_{\mathrm{c}}, \mathrm{J} / \mathrm{kg} \cdot \mathrm{K}$ & $-(8.8 \pm 0.5)$ & & & \\
\hline$\left(\Delta \mathrm{S}_{\mathrm{PCE}}\right)_{\mathrm{cer}}, \mathrm{J} / \mathrm{kg} \cdot \mathrm{K}$ & & $-(3.8 \pm 0.4)$ & & \\
\hline$\left(\Delta \mathrm{T}_{\mathrm{AD}}\right)_{\mathrm{BCE}}, \mathrm{K}$ & $+(1.5 \pm 0.1)$ & $+(1.1 \pm 0.1)$ & $+(0.80 \pm 0.09 *$ & $+(0.79 \pm 0.09)^{*}$ \\
\hline$\left(\Delta \mathrm{T}_{\mathrm{AD}}\right)_{\mathrm{a}}, \mathrm{K}$ & $-(0.34 \pm 0.04)$ & & & \\
\hline$\left(\Delta \mathrm{T}_{\mathrm{AD}}\right)_{\mathrm{b}}, \mathrm{K}$ & $+(0.91 \pm 0.07)$ & & & \\
\hline$\left(\Delta \mathrm{T}_{\mathrm{AD}}\right)_{\mathrm{c}}, \mathrm{K}$ & $+(2.0 \pm 0.1)$ & & & \\
\hline$\left(\Delta \mathrm{T}_{\mathrm{AD}}\right)_{\mathrm{cer}}, \mathrm{K}$ & & $+(0.90 \pm 0.06)$ & & \\
\hline
\end{tabular}

Analysis of the heat capacity $[19,26]$ and linear thermal expansion coefficients [22] of the TGS single crystal within the Pippard equation made it possible to determine the phase transition temperature shifts under uniaxial pressures along the main crystallographic axes, $\mathrm{dT}_{0} / \mathrm{d}_{\mathrm{i}}$ (Table 2 ) : In this case, the volumetric baric coefficient, $\left(\mathrm{dT}_{0} / \mathrm{dp}\right)_{\text {calc }}=\Sigma \mathrm{dT}_{0} / \mathrm{d}_{\mathrm{i}}=19 \mathrm{~K} / \mathrm{GPa}$, agrees with the experimental value $\left(\mathrm{dT}_{0} / \mathrm{dp}\right)_{\exp }$, which confirms the validity of the approach for determining linear baric coefficients. 
The values of the phase transition temperature shift under uniaxial pressure for the studied ceramic and nanocomposite materials were estimated in the framework of the Ehrenfest equation $\left(\mathrm{dT}_{0} / \mathrm{d} \sigma\right)_{\mathrm{cer}}=\mathrm{T}_{0}\left(\Delta \alpha / \Delta \mathrm{C}_{\mathrm{p}}\right)$ using data on the coefficient of linear thermal expansion, $\alpha$, and heat capacity [26].

The calculated linear baric coefficient for quasi-ceramics, $\left(\mathrm{dT}_{0} / \mathrm{d} \sigma\right)_{\mathrm{cer}}$, was found to be very close to the volumetric value, $\mathrm{dT}_{0} / \mathrm{dp}$, determined experimentally (Table 2 ). This means that the value $\left(\mathrm{dT}_{0} / \mathrm{dp}\right)_{\text {calc }}=3\left(\mathrm{dT}_{0} / \sigma\right)_{\text {cer }}$ for this sample determined in the approximation of the isotropic thermal expansion of ceramics turned out to be unreasonably large. One might think that in such a case the equality $\beta=3 \alpha$, where $\alpha$ is measured along the diameter of the pellet, is quite approximate [1]. Indeed, the existence of a texture in the sample cannot be ruled out, which is expressed in the preferred orientation of the crystallites on the pellet surface. This effect was observed in the study of TGS embedded in porous $\mathrm{Al}_{2} \mathrm{O}_{3}$ [37].

As regards nanocomposites, three circumstances must be taken into account. First, an experimentally determined coefficient of linear thermal expansion, $\alpha$, characterizes the expansion of a system consisting of two mechanically interacting components [26]. Secondly, the presence of texture in these samples also cannot be ruled out. Thirdly, to determine the expansion coefficients of the introduced component, changes in its linear/volumetric sizes should be attributed to the sizes of TGS. In this case, the linear baric coefficient, $\left(\mathrm{dT}_{0} / \mathrm{d} \sigma\right)_{\mathrm{cer}}$, for nanocomposites is quite small compared to the value for quasi-ceramics, but the shift of $\mathrm{T}_{0}$ under hydrostatic pressure, $\left(\mathrm{dT}_{0} / \mathrm{dp}\right)_{\text {calc }}$, is close to the experimental data (Table 2).

Using information on the heat capacity, entropy, and thermal expansion [19$21,26]$, as well as the data obtained in this paper on the sensitivity to hydrostatic and 
uniaxial pressure, we determined the nature of the influence of the dimensional factor on the baro- and piezo-caloric properties of ferroelectric TGS in three modifications: single crystal, quasi-ceramics and active component in TGS + PG nanocomposites.

We applied the analysis of the total entropy, $\mathrm{S}(\mathrm{T})$, of the samples consisting of lattice, $\mathrm{S}_{\mathrm{LAT}}(\mathrm{T})$, and anomalous, $\Delta \mathrm{S}(\mathrm{T})$, contributions, which previously allowed us to determine $\mathrm{BCE}$ and PCE in various ferroelastics [27], ferroelectrics [32], and ferromagnets [30]. For this aim, on the temperature dependences $\mathrm{S}(\mathrm{T})$ at different pressures, $\Delta \mathrm{S}(\mathrm{T})$ is shifted along $\mathrm{S}_{\mathrm{LAT}}(\mathrm{T})$ in accordance with the value and sign of the baric coefficient $\mathrm{dT}_{0} / \mathrm{dp}$ (or $\mathrm{dT}_{0} / \mathrm{d} \sigma$ for uniaxial pressure). We believe that the entropy of the phase transition in TGS remains unchanged under pressure. This assumption is supported by preservation of the symmetries of the initial and distorted phases up to the pressure of the triple point, $\mathrm{p} \approx 2.4 \mathrm{GPa}$, discovered recently [20]. Thus, it is unlikely that the low pressure used in our experiments can affect the degree of disordering/ordering of dipoles in both phases and, as a result, the entropy of the $\mathrm{P} 2{ }_{1} / \mathrm{m}$ $\leftrightarrow \mathrm{P} 21_{1}$ transformation

At the first stage, the phase diagram $S(T, p)$ of the quasi-ceramic TGS was analyzed, taking into account the experimentally determined baric coefficient, $\mathrm{dT}_{0} / \mathrm{dp}=$ 23.5 K/GPa (Fig. 3a). Since the changes in entropy under pressure are very small ( $\sim 5$ $\mathrm{J} / \mathrm{kg} \cdot \mathrm{K})$ compared with total entropy $(\sim 1000 \mathrm{~J} / \mathrm{kg} \cdot \mathrm{K})$, the results obtained are shown in both wide and narrow temperature ranges.

The behavior and values of extensive $\mathrm{BCE}, \Delta \mathrm{S}_{\mathrm{BCE}}$, at different pressures was found as a difference $\Delta \mathrm{S}_{\mathrm{BCE}}=\mathrm{S}(\mathrm{T} ; \mathrm{p})-\mathrm{S}(\mathrm{T} ; \mathrm{p}=0)$ at constant temperature (Fig. 3b). By analyzing the plots $S(T ; p)=S_{\mathrm{LAT}}(\mathrm{T} ; \mathrm{p}=0)+\Delta \mathrm{S}(\mathrm{T}, \mathrm{p})$ at constant entropy, $\mathrm{S}(\mathrm{T} ; \mathrm{p})=\mathrm{S}(\mathrm{T}+$ 
$\Delta \mathrm{T}_{\mathrm{AD}}, \mathrm{p}=0$ ), the temperature dependences of the intensive $\mathrm{BCE}$ were established (Fig. 3c).

Fig, $3 \mathrm{~b}$ and $3 \mathrm{c}$ demonstrate that both barocaloric parameters of a quasiceramic sample are significantly lower than those in ferroics considered to be promising solid state refrigerants $[4,7]$.The analysis showed that in order to achieve the highest value of the extensive $\mathrm{BCE}$ equal to the entropy of the phase transition, $\Delta \mathrm{S}_{\mathrm{BCE}}{ }^{\mathrm{MAX}} \approx 20$ $\mathrm{J} / \mathrm{kg} \cdot \mathrm{K}$, very high hydrostatic pressure, $\mathrm{p}>10 \mathrm{GPa}$, is required. One of the main reasons for this phenomenon is associated with a rather small shift in the temperature of the phase transition under hydrostatic pressure,

Due to the close values of $\Delta \mathrm{S}$ and $\mathrm{dT}_{0} / \mathrm{dp}$, the barocaloric effect in single-crystal TGS will be almost the same as in ceramics. However, as mentioned above, the linear baric coefficients for a TGS single crystal are characterized by both different values and a sign, and one of them, namely $\mathrm{dT}_{0} / \mathrm{d}_{\mathrm{c}}=+39 \mathrm{~K} / \mathrm{GPa}$, is one and a half times higher than the volume baric coefficients for both single-crystal and quasi-ceramic samples (Table 2). As a result, the extensive and intensive piezocaloric parameters associated with the pressure along the $c$ axis will exceed the barocaloric parameters of the bulk TGS at the same pressure (Fig. 3 d, e, f). Table 2

Since the baric coefficients of volumetric TGS are not much higher than for TGS+PG nanocomposites, the barocaloric parameters of the latter, referred only to the active component, will decrease slightly (Table 2 ).. However, due to the fact that the entropy of the composites is the sum of the entropies of the components (PG matrix and TGS $), S^{\text {comp }}=S^{\mathrm{PG}}+\left(\mathrm{S}_{\mathrm{LAT}}{ }^{\mathrm{TGS}}+\Delta \mathrm{S}^{\mathrm{TGS}}\right)$, part of the heat released/absorbed by the active component will also be spent on heating/cooling of the PG matrix when applying and removing pressure. It was found that at the same pressure, the barocaloric parameter of 
the studied nanocomposites as a whole, $\left(\Delta \mathrm{T}_{\mathrm{AD}}\right)_{\mathrm{BCE}}=0.34 \mathrm{~K}$ is approximately $70 \%$ lower than for quasi-ceramic TGS (Table 2).

Nevertheless, the obtained results for composites indicate that BCE in the active component is not significantly suppressed under conditions of restricted geometry. This circumstance allows us to consider that the incorporation into a porous glass of a larger amount of the active component with more significant values of both the entropy of the phase transition and the shift of the transformation temperature under pressure will allow one to achieve worthy of attention barocaloric effects in the composite as a whole. In this case, the nanocomposite can be designed in the form of a solid-state refrigerant element more convenient for practical applications in comparison with a single crystal and quasi-ceramics.

It was also of interest to obtain information about the impact of the size factor on the electrocaloric properties of TGS. To this end, we intended to perform direct measurements of the intensive $\mathrm{ECE}\left(\triangle \mathrm{T}_{\mathrm{AD}}\right)_{\mathrm{ECE}}$, in the samples under study, using the technique that we used earlier in the study of ECE in a single-crystal TGS [21]. Unfortunately, we did not succeed in studying quasi-ceramics and the TGS+PG46 nanocomposite. In the first case, the sample showed very strong conductivity due to the high porosity caused by the forced absence of firing after pressing. Changes in the temperature of the TGS + PG46 nanocomposite in the electric field turned out to be below the sensitivity limits of the tech nique used. Therefore, here we present the results of the ECE study only for the TGS+PG320 nanocomposite.

At the first stage, for further characterization of the sample, studies of the heat capacity and permittivity were carried out. 
Fig. 4a shows the temperature dependence of the heat capacity of a sample. The peak on the curve $C_{p}(T)$ associated with the phase transition was observed at $T_{0}=$ $320.5 \pm 1.5 \mathrm{~K}$, which is in good agreement with the data of previous studies of a nanocomposite of the same composition [26].

In order to determine the entropy of the phase transition, the heat capacity of TGS+PG320 nanocompopsite, $\mathrm{C}_{\mathrm{p}}(\mathrm{T})$, was considered as the sum $\mathrm{C}_{\mathrm{p}}(\mathrm{T})=\Delta \mathrm{C}_{\mathrm{p}}(\mathrm{T})+$ $\mathrm{C}_{\text {reg }}(\mathrm{T})$. Here $\Delta \mathrm{C}_{\mathrm{p}}(\mathrm{T})$ is the anomalous heat capacity referring to the TGS component and $\mathrm{C}_{\text {reg }}$ is the regular contribution. The behavior of $\mathrm{C}_{\mathrm{reg}}(\mathrm{T})$ was determined by smoothed interpolation of the $\mathrm{C}_{\mathrm{p}}(\mathrm{T})$ data far above and below the phase transition region by polynomial function and is shown as dashed line in Fig. 4a.

Integration of the function $\left(\Delta \mathrm{C}_{\mathrm{p}} / \mathrm{T}\right)(\mathrm{T})$ showed that the excess entropy, $\Delta \mathrm{S}_{0}=$ $6.0 \pm 0.5 \mathrm{~J} / \mathrm{mol} \cdot \mathrm{K}$ (Insert in Fig.4a), is the same as that found previously for a nanocomposite of the same type [26]. The high reproducibility of the thermodynamic parameters, $\mathrm{T}_{0}$ and $\Delta \mathrm{S}_{0}$, from sample to sample indicates the reliability of the method of preparing nanocomposites TGS + PG.

In Fig. 4b, one can see the temperature dependencies of the permittivity, $\varepsilon$, measured at two frequencies, $1 \mathrm{kHz}$ and $1 \mathrm{MHz}$, Anomaly $\Delta \varepsilon(\mathrm{T})$ related to the phase transition is most pronounced at a high frequency. However, the values of $\Delta \varepsilon(\mathrm{T}) \leq 1$ at both frequencies are very small and comparable with the value mentioned above for TGS+PG composites placed in pentane for pressure measurements. A rather strong increase in $\varepsilon(\mathrm{T})$ at $\mathrm{f}=1 \mathrm{kHz}$ above $\mathrm{T}_{0}$ is, most likely, associated with an increase in the number of mobile charges moving together with the measuring electric field. With an increase in the field frequency, this movement becomes more difficult, and at a frequency of $1 \mathrm{MHz}$ the dielectric constant increases more slowly. 
Thus, in the studied temperature and frequency range, active component of the TGS + PG320 composite can be considered as ferroelectrics with a large leakage current.

It should be noted that there is a fairly large difference between the temperatures of the peak positions on the dependencies $C_{p}(T)\left(T_{\max }{ }^{C p}=320.5 \pm 1.5 \mathrm{~K}\right)$ and $\varepsilon(\mathrm{T})\left(\mathrm{T}_{\max }{ }^{\varepsilon}=329.0 \pm 1.5 \mathrm{~K}\right)\left(\right.$ Fig. 4). However $\mathrm{T}_{\max }{ }^{\varepsilon}$ is in good agreement with the inflection temperature, $\mathrm{T}_{\mathrm{infl}} \approx 328 \mathrm{~K}$, at an anomalous heat capacity above $\mathrm{T}_{\max }{ }^{\mathrm{Cp}}$. The reason is that, $\mathrm{T}_{\mathrm{infl}}$ can be considered as the temperature of the smeared heat capacity jump associated with the phase transition.

Measurements of ECE in the TGS+PG320 nanocomposite were performed in the temperature range $300-330 \mathrm{~K}$.

At the first stage, the temperature drift of the sample was regulated to obtain an optimum rate of the temperature change, $\mathrm{dT} / \mathrm{dt}= \pm 2.5 \cdot 10^{-4} \mathrm{~K} / \mathrm{min}$. A small constant rate of change in temperature of the sample at the absence of electric field, $E=0$, indicates the realization of quasi-adiabatic conditions $(\mathrm{dS} \approx 0)$. At some moment electric field was switched on and then after several minutes was switched off. In this case, an abrupt increase/decrease in the temperature of the sample, $\Delta \mathrm{T}_{\mathrm{AD}}$, should be detected as it was observed, for example, in the study of ECE in a single crystal of TGS [21]. However, the plots $\mathrm{T}(\mathrm{t})$ do not show any significant change in temperature associated with ECE.

Fig. 5 presents some results of measurements of the time dependence of temperature near phase transition in the process of switch on/off electric field: $(E=0)$ $\rightarrow(\mathrm{E}=11.5 \mathrm{kV} / \mathrm{cm}) \rightarrow(\mathrm{E}=0)$ 
Only very small values of experimentally observed changes in temperature, $\Delta \mathrm{T}_{\mathrm{AD}}{ }^{\mathrm{EXP}} \leq 10^{-3} \mathrm{~K}$, related to the system sample+measuring cell+ApiezonN was detected. The process $\mathrm{E}=0$ ends at point 2, where the voltage is applied to the composite electrodes and, as a result, in the process $2-3-4$ there is a strong increase in the rate of temperature change of the sample under the electric field, $\mathrm{dT} / \mathrm{dt} \approx 5 \cdot 10^{-4}$ $\mathrm{K} / \mathrm{min}$

After switching off $\mathrm{E}$, the value of dT/dt was again decreased (process $4-5$ ). These phenomena, which were also observed in some other ferroelectrics $[21,38]$, allowed us to conclude that the strong increase in the heating rate at $\mathrm{E} \neq 0$ is associated, in particular, with the release of Joule heat due to the rather high electric conductivity of the TGS+PG320 nanocomposite. Moreover, as can be seen in Fig. 5, the $\mathrm{E} \neq 0$ process consists of two parts that differ in the dT/dt derivative. In the section $2-3$, the speed is higher, since here the sample is heated both due to ECE and the Joule heat. At point 3, the heat release due to ECE in the TGS component ends and the further temperature increase in the $3-4$ process is associated only with the heat release on the electrical resistance of the nanocomposite.

The adiabatic change in the temperature of the TGS+PG320 composite, $\left(\Delta \mathrm{T}_{\mathrm{AD}}{ }^{\mathrm{ON}}\right)^{\mathrm{EXP}}=\left(\Delta \mathrm{T}_{\mathrm{AD}}{ }^{\mathrm{OFF}}\right)^{\mathrm{EXP}} \approx 5 \cdot 10^{-4} \mathrm{~K}$ (Fig. 5), observed when applying/removing an electric field of fairly high intensity, $\mathrm{E}=11.5 \mathrm{kV} / \mathrm{cm}$, was three orders of magnitude less than $\Delta \mathrm{T}_{\mathrm{AD}}=0.15 \mathrm{~K}$ for a single-crystal TGS at a lower field, $\mathrm{E}=2.8 \mathrm{kV} / \mathrm{cm}[21]$.

Such a large difference in $\Delta \mathrm{T}_{\mathrm{AD}}$ cannot be explained only by the fact that the change in the energy of the active ferroelectric component associated with ECE during an adiabatic change in the electric field is spent on heating/cooling not only TGS, but also PG, measuring cell, and ApiezonN. Evaluation of the actual $\Delta \mathrm{T}_{\mathrm{AD}}$ in the TGS 
component, taking into account the ratio between the heat capacities of the parts of the system under study, shows that this value does not exceed $510^{-3} \mathrm{~K}$.

Another reason may be due to poor contact between the embedded TGS and the electrodes on the surface of the TGS+PG320 composite. In this case, the observed $\Delta \mathrm{T}_{\mathrm{AD}}$ may be associated with a small part of the active component located in the electric field.

The third reason relates to the issue of the influence of the size of TGS crystallites. Recent studies of ECE in nanoceramic $\mathrm{BaTiO}_{3}$ with a particle size of 100$400 \mathrm{~nm}$ showed a strong decrease in $\Delta \mathrm{T}_{\mathrm{AD}}$ compared to single crystal and microceramics [39].

And finally, the fourth reason can be related both to the texture of the embedded TGS observed in [37] and to the random orientation of nanocrystallites in the pores of the matrix. Since TGS is a uniaxial ferroelectric, both phenomena can lead to a significant decrease in the electrocaloric response in nanocomposites. It can be confidently believed that, for the same reason, a strong decrease in the anomalous permittivity was observed.

\section{Conclusions}

We performed studies of the effect of hydrostatic pressure, size factor, and electric field on the ferroelectric phase transition $\mathrm{P} 2{ }_{1} \leftrightarrow \mathrm{P} 2{ }_{1} / \mathrm{m}$ in bulk quasi-ceramic TGS and nanocomposites consisting of TGS embedded in porous glasses, TGS+PG320 and TGS+PG46.

The size factor insignificantly affects the sensitivity of ferroelectric TGS in various modifications to changes in external hydrostatic pressure. A small sequential 
decrease in the baric coefficient is found in the materials under study $\left(\mathrm{dT}_{0} / \mathrm{dp}, \mathrm{GPa} / \mathrm{K}\right)$ : 26 (single-crystal TGS [20,35,36]), $23.5 \pm 1.5$ (quasi-ceramic TGS), $19.8 \pm 1.5$ (TGS+PG320), 19.5 $\pm 1.5($ TGS+PG46)

An analysis of the phase diagrams $S(T, p)$, taking into account the experimentally determined baric coefficients, made it possible to determine the extensive and intensive barocaloric parameters under isothermal and adiabatic conditions, respectively. The BCE in active component of the TGS+PG nanocomposites is comparable with the barocaloric characteristics of the bulk TGS, which means that there is no suppression of the effect under conditions of restricted geometry. This experimental fact suggests the possibility of designing a promising working element for solid-state cooling based on BCE in a nanocomposite with an active component with large values of the entropy of the phase transition and the baric coefficient.

The significant anisotropy of the thermal expansion of single-crystal TGS is the reason for the large linear baric coefficient along the $c$ axis, $\mathrm{dT}_{0} / \mathrm{d}_{\mathrm{c}}=+39 \mathrm{~K} / \mathrm{GPa}$, which exceeds the volumetric baric coefficient by a factor of 1.5. As a result, at the same pressure, the intensive and extensive PCE is significantly larger than the $\mathrm{BCE}$ parameters.

A strong decrease in both the anomalous permittivity and the electrocaloric effect in the ferroelectric component of the TGS+PG320 nanocomposite was found in comparison with the single-crystal TGS.

Among a number of reasons for this phenomenon considered in this paper, the most likely one is related to the texture of the embedded TGS and with the random orientation of nanocrystallites in the pores of the matrix. 


\section{Declaration of competing interest}

The authors declare that they have no known competing financial interests or personal relationships that could have appeared to influence the work reported in this paper.

Funding: This research did not receive any specific grant from funding agencies in the public, commercial, or not-for-profit sectors.

\section{References}

[1] L. Mañosa, A. Planes. Materials with giant mechanocaloric effects: cooling by strength. Advanced Materials 29 (2017) 1603607

[2] A.M. Tishin, Y.I. Spichkin, The magnetocaloric effect and its applications, Institute of Physics Publishing, London, 2003.

[3] K.A.Jr. Gschneidner, V.K. Pecharsky, A.O. Tsokol, Recent developments in magnetocaloric materials, Rep. Prog. Phys. 68 (2005) 1479. https://doi.org/10.1088/0034-4885/68/6/r04

[4] L. Mañosa, A. Planes, M. Acet, Advanced materials for solid-state refrigeration, J. Mater. Chem. A 1 (2013) 4925-4936. https://doi.org/10.1039/C3TA01289A

[5] M. Ožbolt, A. Kitanovski, J. Tušek, A. Poredoš, Electrocaloric refrigeration: thermodynamics, state of the art and future perspectives, Int. J. Refrig. 40 (2014) 174188. https://doi.org/10.1016/j.ijrefrig.2013.11.007

[6] I.N. Flerov, M.V. Gorev, A. Tressaud, N.M. Laptash, Perovskite-like fluorides and oxyfluorides: phase transitions and caloric effects Crystallography Rep. 56 (2011) 9-17. https://doi.org/10.1134/S106377451101010X 
[7] I.N. Flerov, E.A. Mikhaleva, M.V. Gorev, A.V. Kartashev, Caloric and multicaloric effects in oxygen ferroics and multiferroics, Phys. Solid State 57 (2015) 429-441. https://doi.org/10.1134/S1063783415030075

[8] A. Cizman, T. Antropova, I. Anfimova, I. Drozdova, E. Rysiakiewicz-Pasek, E.B. Radojewska, R. Poprawski, Size-driven ferroelectric-paraelectric phase transition in TGS nanocomposites, J. Nanopart. Res. 15 (2013) 1807. https://doi.org/10.1007/s11051-013-1807-y

[9] S.V. Baryshnikov, A.Yu. Milinskiy, E.V. Charnaya, A.S. Bugaev, M.I.

Samoylovich, Dielectric studies of ferroelectric $\mathrm{NH}_{4} \mathrm{HSO}_{4}$ nanoparticles embedded into porous matrices, Ferroelectrics 493 (2016) 85-92. https://doi.org/10.1080/00150193.2016.1134174

[10] E. Rysiakiewicz-Pasek, R. Poprawski, J. Polanska, A. Urbanowicz, A. Sieradzki, Properties of porous glasses with embedded ferroelectric materials, J. Non-Cryst. Solids 352 (2006) 4309-4314 https://doi.org/10.1016/j.jnoncrysol.2006.07.026

[11] V. Tarnavich, L. Korotkov, O. Karaeva, A. Naberezhnov, E. Rysiakiewicz-Pasek, Effect of restricted geometry on structural phase transitions in $\mathrm{KH}_{2} \mathrm{PO}_{4}$ and $\mathrm{NH}_{4} \mathrm{H}_{2} \mathrm{PO}_{4}$ crystals, Optica Applicata, 40 (2010) 305-309.

[12] M. Kinka, J. Banys, A. Naberezhnov, Dielectric properties of $\mathrm{NaNO}_{2}$ and $\mathrm{NaNO}_{3}$ confined in porous glass. Ferroelectrics, 390 (2009) 160-167. https://doi.org/10.1080/00150190903000807 [13] V.V. Tarnavich, A.S. Sidorkin, T.N. Korotkova, E. Rysiakiewicz-Pasek, L.N. Korotkov, N.G. Popravko, "Restricted geometry" effect on phase transitions in KDP, ADP, and CDP nanocrystals, Crystals 9 (2019) 593.

https://doi.org/10.3390/cryst9110593 
[14] N.G. Popravko, A.S. Sidorkin, S.D. Milovidova, O.V.Rogazinskaya, Structure and electrical properties of nancomposites with TGS inclusions. Ferroelectrics 443 (2013)

8-15. https://doi.org/10.1080/00150193.2013.778577

[15] S.D. Milovidova, A.S. Sidorkin, O.V. Rogazinskaya, E. V. Vorotnikov, Dielectric properties of the mixed nanocomposites: Triglycine sulfate - silica. Ferroelectrics 497 (2016) 69-73. https://doi.org/10.1080/00150193.2016.1162620

[16] Z. Kutnjak, B. Vodopivec, R. Blinc, A.V. Fokin, Yu.A. Kumzerov, S.B.

Vakhrushev, Calorimetric and dielectric studies of ferroelectric sodium nitrite confined in a nanoscale porous glass matrix, J, Chem. Phys. 123 (2005) 084708.

https://doi.org/10.1063/1.2007687

[17] Yu.A. Kumzerov, N.F. Kartenko, L.S. Parfen’eva, I.A. Smirnov, A.V. Fokin, D. Wlosewicz, H. Misiorek, A. Jezowski, Capacity and thermal conductivity of a nanocomposite chrysolite asbestos-KDP $\left(\mathrm{KH}_{2} \mathrm{PO}_{4}\right)$, Phys. Solid State, 53 (2011) 1099-1103. https://doi.org/10.1134/S1063783411050167

[18] E.A. Mikhaleva, I.N. Flerov, A.V. Kartashev, M.V. Gorev, M.S. Molokeev, E.V. Bogdanov, V.S. Bondarev, L.N. Korotkov, E. Rysiakiewicz-Pasek, Effect of restricted geometry and external pressure on the phase transitions in ammonium hydrogen sulfate confined in a nanoporous glass matrix, J. Mater Science 53 (2018) 12132-12144. https://doi.org/10.1007/s10853-018-2467-1

[19] B.A. Strukov, E.P. Ragula, S.V. Arkhangel'skaya, I.V. Shnaidshtein, Logarithmic singularity in the specific heat in the vicinity of phase transitions in uniaxial ferroelectrics, Phys. Solid State 40 (1998) 94 - 95. https://doi.org/10.1134/1.1130241 
[20] Y. Kobayashi, S. Sawada, H. Furuta, S. Endo, K. Deguchi, Ferroelectric TGS

$\left(\left(\mathrm{NH}_{2} \mathrm{CH}_{2} \mathrm{COOH}\right)_{3} \cdot \mathrm{H}_{2} \mathrm{SO}_{4}\right)$ under high pressure, J. Phys.: Condens. Matter 14 (2002)

$11139-11142$.

https://doi.org/ 10.1088/0953-8984/14/44/440

[21] V.S. Bondarev, E.A. Mikhaleva, I.N. Flerov, M.V. Gorev, Electrocaloric Effect in triglycine sulfate under equilibrium and nonequilibrium thermodynamic conditions, Phys. Solid State, 59 (2017) 1118-1126. https://doi.org/10.1134/S1063783417060051

[22] T. Yamaguchi, N. Nakatani, T. Kikuta, T. Kurihama, T. Mitsui, Y. Seimiya. S. Yoshizava, F. Shimizu, M. Takashige, Dilatometric study on monoclinic crystals of ferroelectric TGS down to cryogenic temperature region, Ferroelectrics 337 (2006) 5969. https://doi.org/10.1080/00150190600716135

[23] M.I. Kay, R. Kleinberg, The crystal structure of triglycine sulfate, Ferroelectrics 5 (1973) 45-52.

[24] F.H. Allen, The Cambridge structural database: a quarter of a million crystal structures and rising, Acta Crystallogr. B58 (2002) 380-388.

[25] R.R. Choudhury, R Chitra, Single crystal neutron diffraction study of triglycine sulphate revisited, Pramana - J. Phys. 71 (2008) 911-915.

[26] E.A. Mikhaleva, I.N. Flerov, A.V. Kartashev, M.V. Gorev, M.S. Molokeev, L.N. Korotkov, E. Rysiakiewicz-Pasek, Specific heat and thermal expansion of triglycine sulfate-porous glass nanocomposites, Phys. Solid State 60 (2018) 1338-1343. https://doi.org/10.1134/S1063783418070181

[27] M.V. Gorev, E.V. Bogdanov, I.N. Flerov. T - p phase diagrams and barocaloric effect in materials with successive phase transitions, J. Phys D: Appl. Physics 50 (2017) 384002. https://doi.org/10.1088/1361-6463/aa8025 
[28] Bruker AXS TOPAS V4 (2008) General profile and structure analysis software for powder diffraction data-user's manual.

Bruker AXS, Karlsruhe.

[29] A. Fokin, Yu. Kumzerov, E. Koroleva, A. Naberezhnov, O. Smirnov, M. Tovar, S. Vakhrushev, M. Glazman, Ferroelectric phase transitions in sodium nitrite nanocomposites, J. Electroceram 22 (2009) 270-275. https://doi.org/10.1007/s10832$\underline{008-9431-4}$

[30] A.V. Kartashev, I.N. Flerov, N.V. Volkov, K.A. Sablina, Adiabatic calorimetric study of the intense magnetocaloric effect and the heat capacity of $\left(\mathrm{La}_{0.4} \mathrm{Eu}_{0.6}\right)_{0.7} \mathrm{~Pb}_{0.3} \mathrm{MnO}_{3}$, Phys. Solid State 50 (2008) 2115 -2120. https://doi.org/10.1134/S1063783408110188

[31] M.V. Gorev, I.N. Flerov, E.V. Bogdanov, V.N. Voronov, N.M. Laptash, Barocaloric effect near the structural phase transition in the $\mathrm{Rb}_{2} \mathrm{KTiOF}_{5}$ oxyfluoride, Phys. Solid State 52 (2010) 377-383. https://doi.org/10.1134/S1063783410020253. [32] M.V. Gorev, E.A. Mikhaleva, I.N. Flerov, E.V.Bogdanov. Conventional and inverse barocaloric effects in ferroelectric $\mathrm{NH}_{4} \mathrm{HSO}_{4}$, J. Alloys Compd. 806 (2019) $1047-1051$.

https://doi.org/10.1016/j.jallcom.2019.07.273

[33] M.V. Gorev, E.V. Bogdanov, I.N. Flerov, Conventional and inverse barocaloric effects around triple points in ferroelastics $\left(\mathrm{NH}_{4}\right)_{3} \mathrm{NbOF}_{6}$ and $\left(\mathrm{NH}_{4}\right)_{3} \mathrm{TiOF}_{5}$, Scripta Materialia 139 (2017) 53-57.

https://doi.org/10.1016/j.scriptamat.2017.06.022

[34] V.S. Bondarev, E.A. Mikhaleva, M.V. Gorev, I.N. Flerov, 
Intensive electrocaloric effect in triglycine sulphate under nonequilibrium thermal conditions and periodic electric field, Physica Status Solidi (B) 253 (2016) 2073 - 2078. https://doi.org/10.1002/pssb.201600339

[35] G.G. Leonidova, I.N. Polandov, I.P. Golentovskaya, Effect of hydrostatic pressure on the phase transition temperature in triglycine sulfate, Sov. Phys. Solid State 4 (1962) 3337-3340.

[36] J. Stankowsky, A, Gałęzewski, S. Waplak, U. Cruszczyńska, H. Gierszal, Dielectric properties of TGS and TGFB monocrystals under high pressure, Ferroelectrics 6 (1974) 209-214. https://doi.org/10.1080/00150197408243969 [37] O.M. Golitsyna, S.N. Drozhdin, I.E. Zanin, A.E. Gridnev, Structure of triglycine sulfate embedded in porous aluminum oxide, Phys. Solid State 54 (2012) 2296-2300. https://doi.org/10.1134/S1063783412110091

[38] E.A. Mikhaleva, I.N. Flerov, V.S. Bondarev, M.V. Gorev, A.D. Vasiliev, T. N. Davydova. Phase transitions and caloric effects in ferroelectric solid solutions of ammonium and rubidium hydrosulfates, Phys. Solid State 53 (2011) 510-517. https://doi.org/10.1134/S1063783411030188

[39] A.V. Kartashev, V.S. Bondarev, I.N. Flerov, M.V. Gorev, E.I. Pogorel’tsev, A.V.

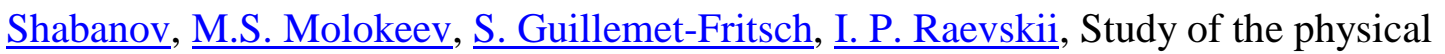
properties and electrocaloric effect in the $\mathrm{BaTiO}_{3}$ nano- and microceramics, $\underline{\text { Phys. Solid }}$ State 61 (2019) 1052-1061. https://doi.org/10.1134/S1063783419060088

\section{Figure Captions}


Figure1. Difference Rietveld plots at room temperature: (a) quasi-ceramic TGS, (b) TGS+PG46, (c) TGS+PG320.

Figure 2. Temperature dependences of anomalous permittivity under pressure at $1 \mathrm{kHz}$ for (a) quasi-ceramic TGS, (b) TGS+PG320, (c) TGS+PG46. (d) T-p phase diagrams of materials under study.

Figure 3. (a) Temperature dependencies of total entropy of quasi-ceramic TGS under different hydrostatic pressure near $\mathrm{T}_{0}$. (b) Barocaloric entropy and (c) adiabatic temperature changes at different hydrostatic pressure. (d) Temperature dependencies of total entropy of single-crystal TGS under different uniaxial pressure along the axis c. (e) Piezocaloric entropy, $\Delta \mathrm{S}_{\mathrm{PCE}}$, and (f) adiabatic temperature changes $\Delta \mathrm{T}_{\mathrm{AD}}$ at different uniaxial pressure in single-crystal TGS.

Figure 4. Experimental data for TGS+PG320. (a) Temperature dependence of the heat capacity. Dashed line is the regular part $\mathrm{C}_{\text {reg. }}$ Inset shows the behavior of the anomalous entropy, $\Delta \mathrm{S}$. (b) Temperature dependences of permittivity at $1 \mathrm{kHz}(1)$ and $1 \mathrm{MHz}(2)$. Figure 5. Time dependence of the temperature of the system (TGS+PG320) + measuring cell + ApiezonN in processes $1-2(\mathrm{E}=0), 2-3-4(\mathrm{E}=11.5 \mathrm{kV} / \mathrm{cm}), 4-5$ $(\mathrm{E}=0)$ near the phase transition temperature. 


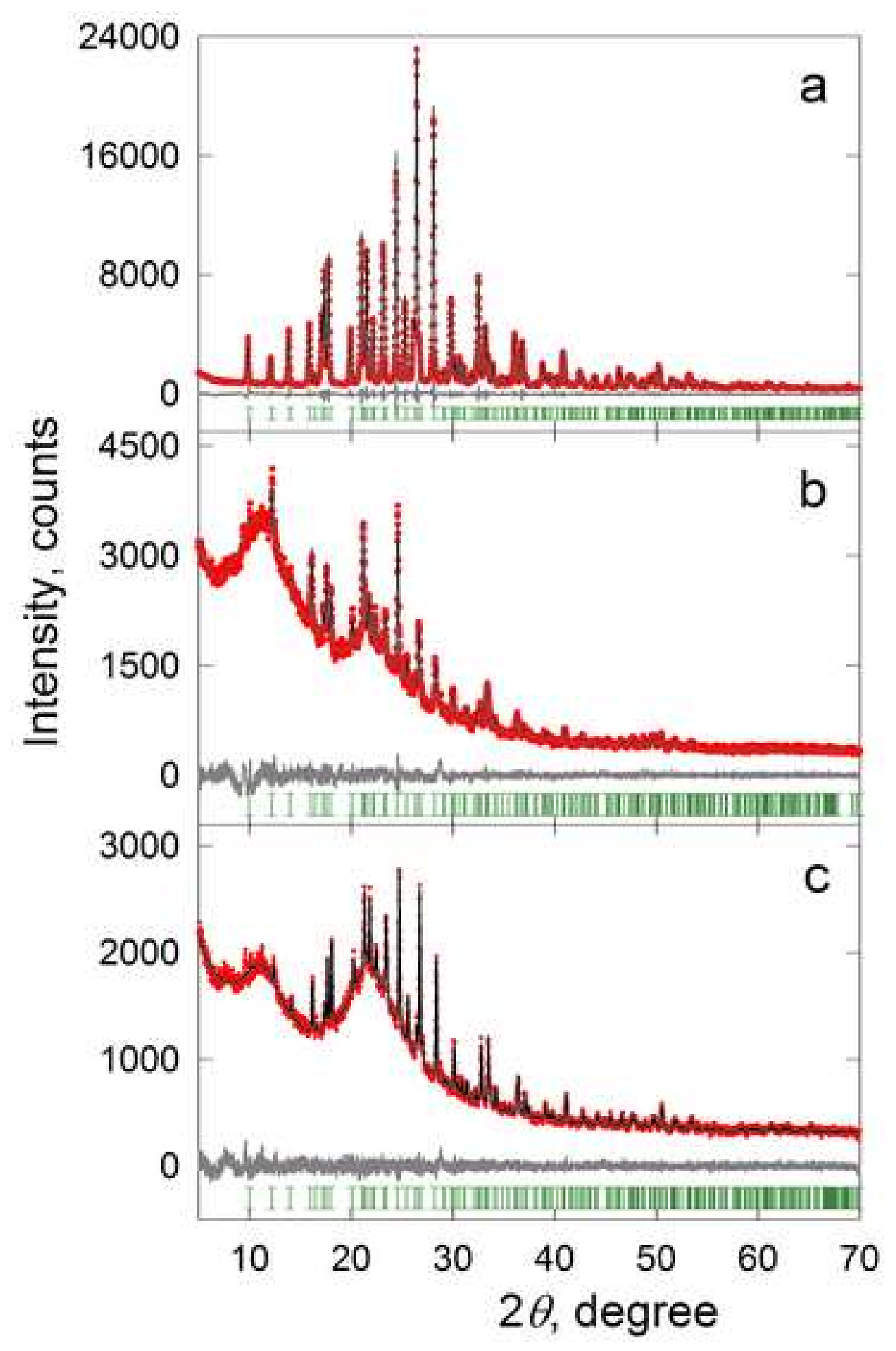



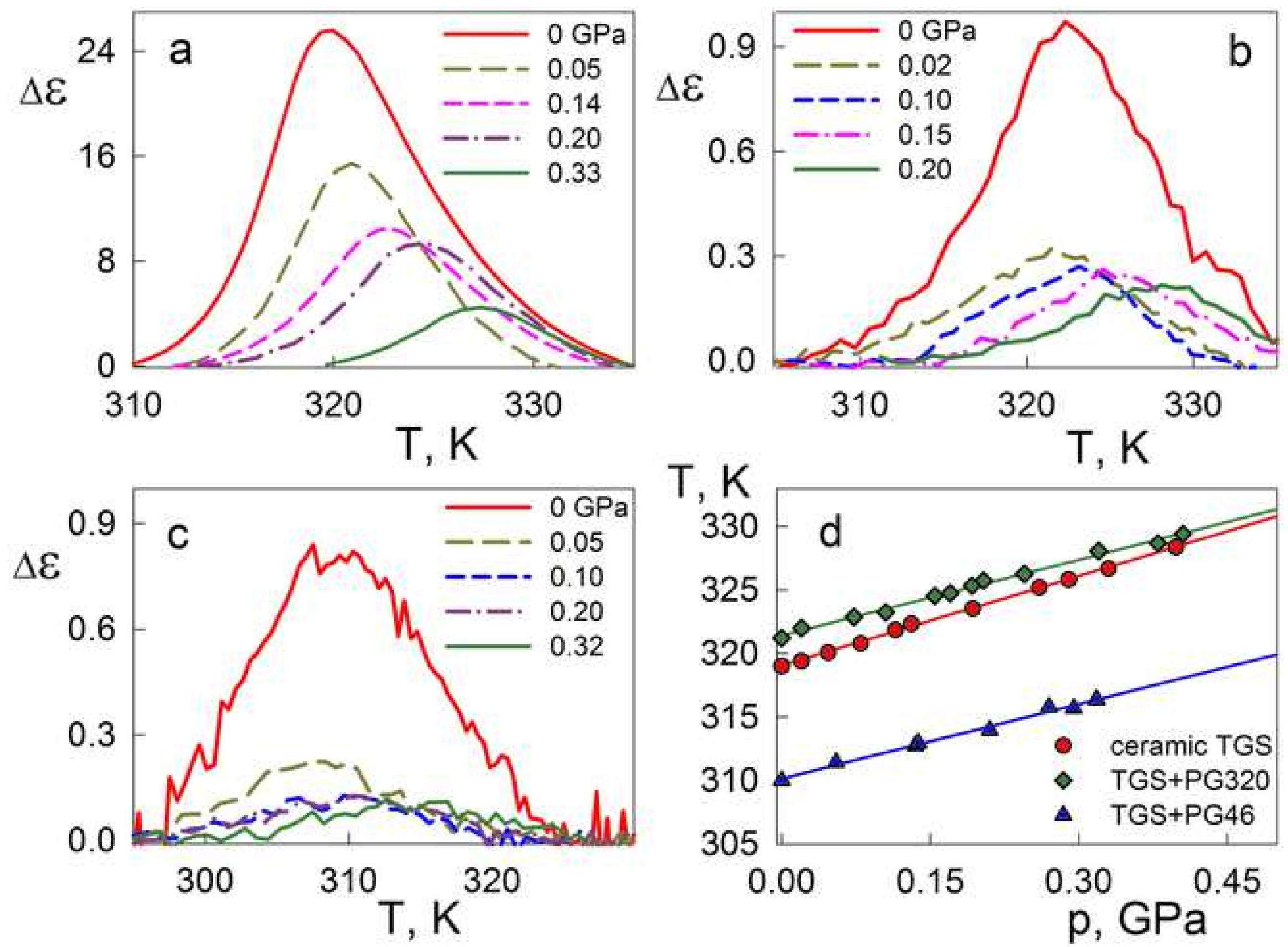

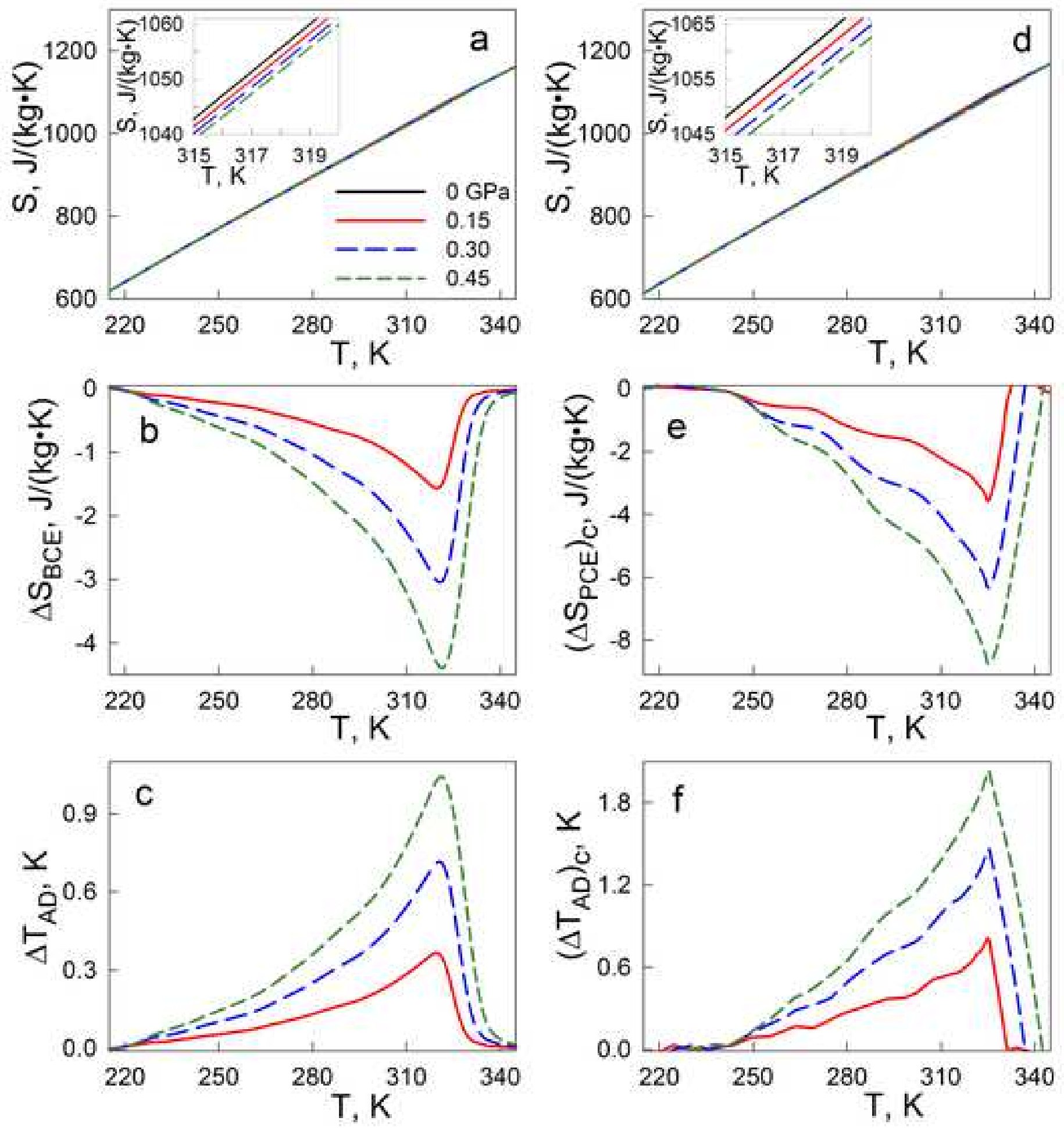

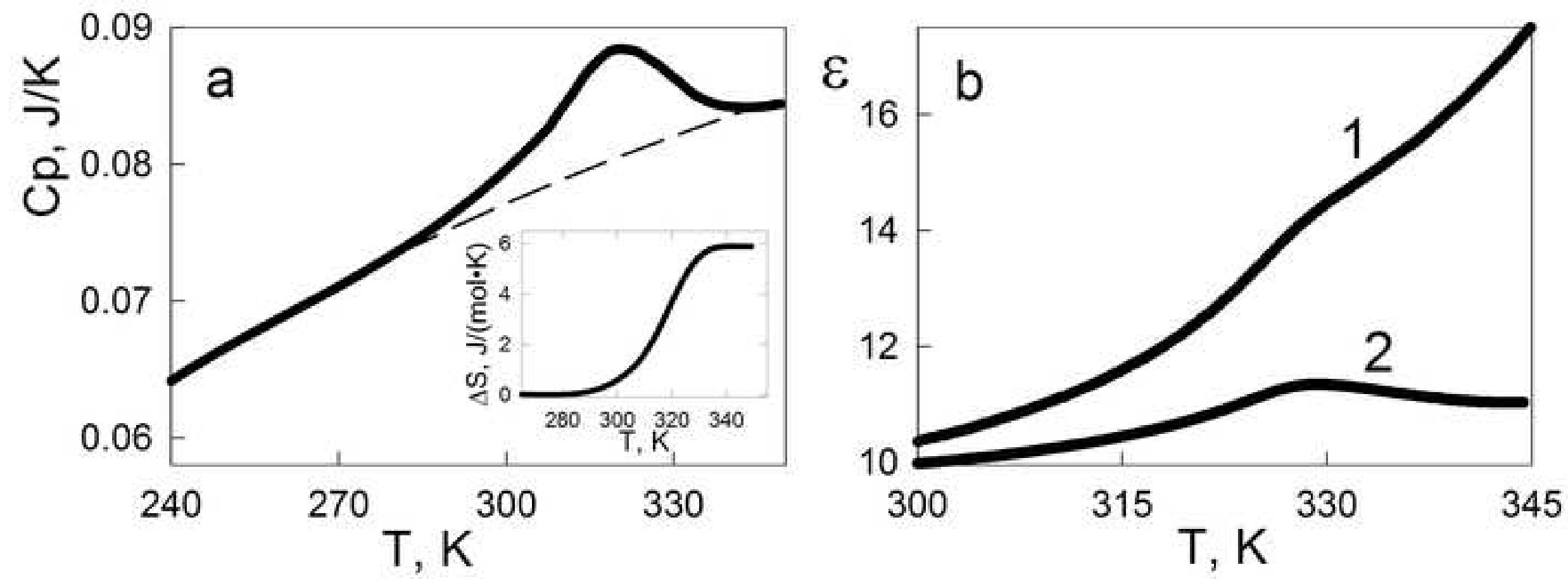


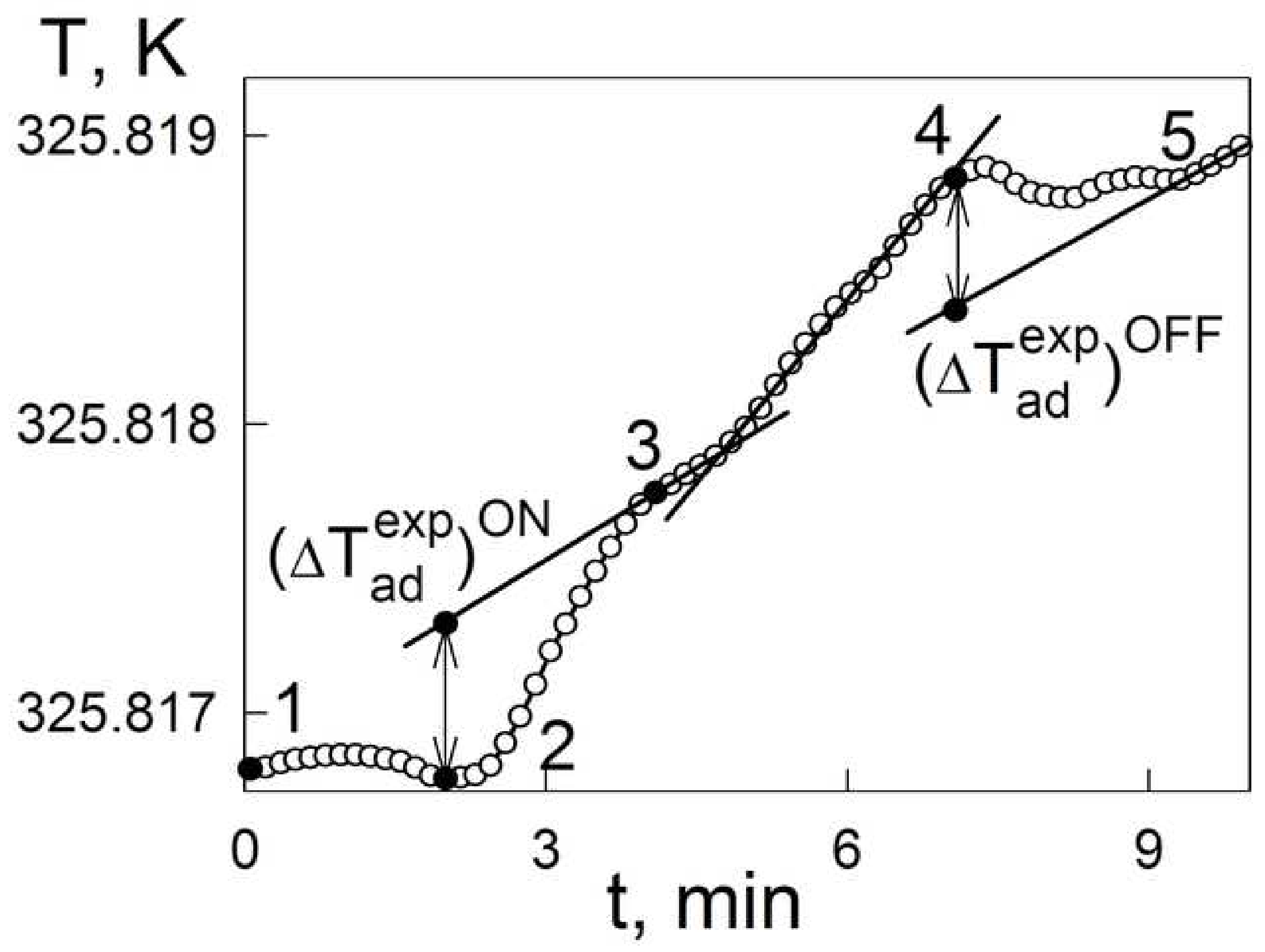




\section{Declaration of interests}

XX The authors declare that they have no known competing financial interests or personal relationships that could have appeared to influence the work reported in this paper.

The authors declare the following financial interests/personal relationships which may be considered as potential competing interests:

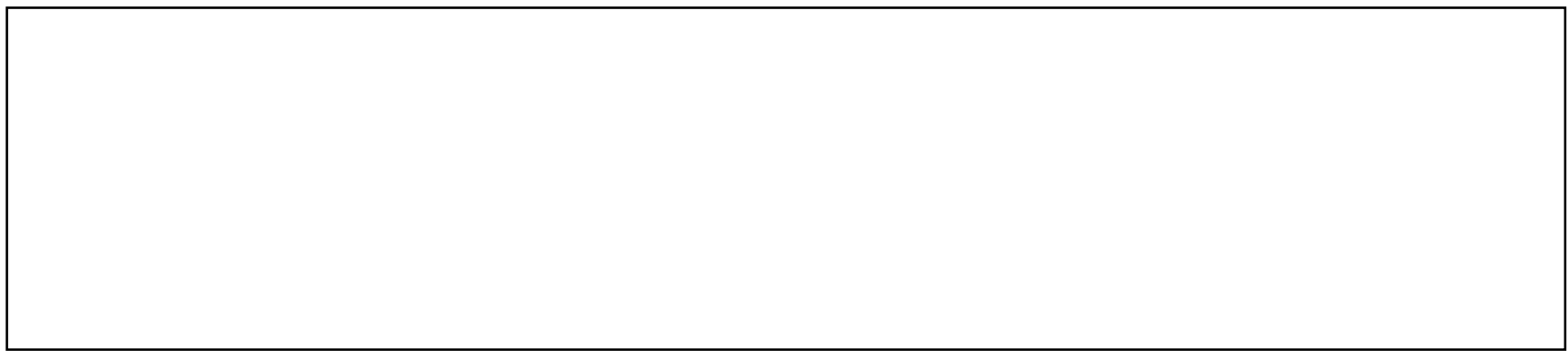

